\title{
On the pressure evolution of dynamic properties of supercooled liquids
}

\author{
Aleksandra Drozd-Rzoska ${ }^{1}$, Sylwester J Rzoska ${ }^{1}$, \\ C Michael Roland ${ }^{2}$ and Attila R Imre ${ }^{3}$ \\ ${ }^{1}$ Institute of Physics, Silesian University, ulica Uniwersytecka 4, 40-007 Katowice, Poland \\ ${ }^{2}$ Naval Research Laboratory, Chemistry Division, Code 6120, Washington, \\ DC 20375-5342, USA \\ ${ }^{3}$ KFKI Atomic Energy Research Institute, 1525 Budapest, POB 49, Hungary
}

Received 4 April 2008

Published 29 May 2008

Online at stacks.iop.org/JPhysCM/20/244103

\begin{abstract}
A pressure counterpart of the Vogel-Fulcher-Tammann (VFT) equation for representing the evolution of dielectric relaxation times or related dynamic properties is discussed: $\tau(P)=\tau_{0}^{P} \exp \left[D_{P} \Delta P\left(P_{0}-\Delta P\right)\right]$, where $\Delta P=P-P_{\mathrm{SL}}, P_{0}$ is the ideal glass pressure estimation, $D_{P}$ is the pressure fragility strength coefficient, and the prefactor $\tau_{0}^{P}$ is related to the relaxation time at the stability limit $\left(P_{\mathrm{SL}}\right)$ in the negative pressure domain. The discussion is extended to the Avramov model (AvM) relation $\tau(T, P)=\tau_{0} \exp \left[\varepsilon\left(T_{\mathrm{g}}(P) / T\right)^{D}\right]$, supplemented with a modified Simon-Glatzel-type equation for the pressure dependence of the glass temperature $\left(T_{\mathrm{g}}(P)\right)$, enabling an insight into the negative pressure region. A recently postulated (Dyre 2006 Rev. Mod. Phys. 78 953) comparison between the VFT and the AvM-type descriptions is examined, for both the temperature and the pressure paths. Finally, we address the question 'Does fragility depend on pressure?' from the title of Paluch $\mathrm{M}$ et al (2001 J. Chem. Phys. 114 8048) and propose a pressure counterpart for the 'Angell plot'.
\end{abstract}

\section{Introduction}

On cooling a liquid to the glass transition a tremendous change in dynamic properties occurs [1-6]. A decade ago it was postulated that [3] 'determining the general behavior of liquids near glass temperature $\left(T_{\mathrm{g}}\right)$ at high pressures is the key problem in the challenging field of viscous liquids and the glass transition'. Indeed, in subsequent years it was shown that many phenomenological and theoretical predictions can be verified only by means of comprehensive temperature and pressure investigations [1, 4-50]. A fundamental prerequisite in such studies is a reliable parameterization of the pressure evolution of the dynamic properties. For their temperature dependence under atmospheric pressure, the Vogel-FulcherTammann (VFT) relation is most often used [1-6, 51]:

$$
\tau(T)=\tau_{0}^{T} \exp \left(\frac{B}{T-T_{0}}\right)=\tau_{0}^{T} \exp \left(\frac{D_{T} T_{0}}{T-T_{0}}\right)
$$

where $D_{T}$ is the fragility strength coefficient and $T_{0}$ is the VFT-based estimate of the ideal glass temperature. Similar dependences can be written for the dielectric (structural) relaxation time $\tau(T)$, viscosity $\eta(T)$, DC conductivity $\sigma(T)$, and diffusion coefficient $d(T)$ [1, 4-10].
It is noteworthy that Johari [52] questioned the validity of the substitution $B=D_{T} T_{0}$, since 'this form does not yield the Arrhenius equation for $T=0 \mathrm{~K}$ '. Despite this objection, the coefficient $D_{T}$ estimated via equation (1) remains one of basic parameters characterizing the fragility of glassy systems [1-6]. The validity of the VFT equation (1) for non-atmospheric isobars $(P \gg 0.1 \mathrm{MPa})$ has also been tested $([4,5]$ and references therein).

A simple extension of equation (1) for portraying both the temperature and the pressure $(P)$ behavior was used by several groups [10, 53-57]:

$$
\tau(T, P)=\tau_{0}^{T} \exp \left(\frac{B^{\prime}+a P}{T-\left(T_{0}+b P\right)}\right) .
$$

However, the linear pressure dependences of $T_{0}(P)$ and $B(P)$, assumed in equation (2), are valid only over a narrow range of pressures, mainly for so-called 'strong' glass formers.

It is worth recalling that in 1967, Greet and Turnbull [58] introduced the following relation for portraying the isothermal pressure behavior of the viscosity of supercooled o-terphenyl:

$$
\eta(T)=\eta_{0}^{P} \exp \left(\frac{B}{P_{0}-P}\right) .
$$


Turnbull et al [58, 59] also showed that the VFT expression can be derived from the Doolittle expression $[1,60]$, namely,

$$
\eta(T, P), \tau(T, P) \propto \exp \left[\frac{B^{\prime}}{f}\right]
$$

where $f=v_{\mathrm{g}} / v_{\mathrm{f}}$ is the fractional free volume, $v_{\mathrm{g}}$ denotes the volume at the glass transition, and $v_{\mathrm{f}}$ is the free volume. The universal value of the coefficient $B^{\prime}=0.9 \pm 0.3$ was suggested [58, 59].

The VFT equation (1) can be obtained assuming $f(T, P=$ const $)=\chi_{T}\left(T-T_{0}\right)[58]$ and equation (3) taking $f(P, T=$ const $)=\alpha_{P}\left(P_{0}-P\right)[59]$, where $\chi_{T}$ and $\alpha_{P}$ denote the isothermal compressibility and thermal expansion coefficient, respectively. In subsequent years, equation (3) was used occasionally, first by Johari and Whalley [61] for glycerol, and much later by Paluch et al [62] for supercooled dibutyl phthalate. However, in 1998 it was noted that it can yield a reliable parameterization only for fragile glass formers [7]. Consequently, a new pressure counterpart of the VFT relation (referred to herein as the PVFT), which introduced the pressure fragility strength coefficient $D_{P}$, was proposed [7]:

$$
\tau(P)=\tau_{0}^{P} \exp \left(\frac{D_{P} P}{P_{0}-P}\right) .
$$

This expression can be used for strong glass formers, because it smoothly approaches the Arrhenius pattern if $P_{0}$ is well above the moderate pressure domain, usually available in experiments. For fragile glass formers the value of $P_{0}$ can approach this range of pressures and then equation (5) coincides with the Turnbull-Johari equation (3). Over the last decade the PVFT equation (5) has become a key tool for portraying the pressure evolution of dynamic properties, including both fundamental and practical issues [4-50].

In the recent review [2] on the glass transition the following expression was promoted as a particularly promising alternative to the VFT equation:

$$
\tau(T)=\tau_{0} \exp \left(C / T^{D}\right) .
$$

This dependence was first proposed in 1931 [63], revisited in 1976 [64] and 1987 [65], and recently obtained as the output of models by Avramov (1998, [66]) and Zhang (2003, [67]). The Avramov model (AvM) made it possible to include both the temperature and the pressure behavior $[66,68,69]$ :

$$
\tau(T, P)=\tau_{0} \exp \left[\varepsilon\left(\frac{T_{\mathrm{g}}(P)}{T}\right)^{\alpha}\right]
$$

where $\varepsilon=E_{\max } / \sigma_{\mathrm{r}}, \sigma_{\mathrm{r}}$ is a dispersion of the reference state with the related entropy, $E_{\max }$ is the maximum allowed energy, $\alpha=2 C_{P} / Z R=D$ is a measure of the fragility, $Z$ is the degeneracy of the system, $C_{P}$ the specific heat.

The AvM assumes that the configurational entropy is the control parameter for the structural relaxation and thus can account for the recently discovered thermodynamic scaling of $\tau(T, P)$ data [34, 35]. However, it was pointed out that equation (7) incorrectly implies that the fragility is independent of pressure [10]. It was also found that the temperature dependence of the half-width of the loss peak as predicted from the Avramov model is at odds with experimental observations [70]. Notwithstanding these problems, the Avramov model [68, 69] yields pressure dependences of the glass temperature which coincide with the empirical expression of Andersson and Andersson (AA) [8], namely [68, 69],

$$
T_{\mathrm{g}}(P)=T_{\mathrm{g}}^{0}\left(1+\frac{P}{\Pi}\right)^{\beta / \alpha}
$$

where $\beta=2 \alpha_{0} V_{\mathrm{m}} \Pi / Z R$ and $V_{\mathrm{m}}$ is the molar volume. The constant $\Pi$ describes the pressure dependence of the thermal expansion coefficient $\alpha_{P}=V^{-1}(\partial V / \partial T)_{P}=\alpha_{0} \Pi /(\Pi+P)$. $T_{\mathrm{g}}^{0}$ and $\alpha_{0}$ are the reference temperature and thermal expansion coefficient at zero pressure.

It is noteworthy that the AA relation has become perhaps the most popular tool for $T_{\mathrm{g}}(P)$ parameterization [3, 5, 12-15, 17-23, 25-35, 37]. In fact, it parallels the Simon-Glatzel (SG) equation, the key expression used for describing the pressure evolution of the melting temperature $\left(T_{\mathrm{m}}(P)\right)$ ([71] and references therein). The ability of the Avramov model to portray $\tau(T, P)$ or $\eta(T, P)$ has been shown in many papers $[10,47,66,68-70,72-74]$. Nevertheless, when discussing the VFT and AvM descriptions of experimental data it is worth recalling the statement from the recent review [2]: 'no systematic analysis has investigated which of [these equations] generally give the best fit of data'.

Herein we present such a comparison, for both the temperature and the pressure paths to the glass transition. Modified forms of the PVFT and AvM relations are proposed, for removing some inherent inconsistencies as well as enabling extension into the negative pressures (isotropically stretched liquid) domain [75-81]. All of this led to a revised answer to the question 'Does fragility depend on pressure?' from the title of [13] and to a proposal of a pressure counterpart of the 'Angell plot' [1-6, 82, 83].

The discussion is based on $\tau(T)$ and $\tau(P)$ experimental data for low molecular weight, van der Waals-type, glassforming liquids: diethyl phthalate [23, 41], propylene carbonate [29] and salol [19]. We do not consider the volumerelated $(\tau(V))$ behavior, for which an extensive discussion can be found in a series of recent papers [34, 35, 84-86].

\section{A pressure counterpart of the VFT relation}

The VFT equation (1) is probably the most commonly used expression for portraying the temperature evolution of the structural ( $\alpha$-, primary, main) relaxation times $\tau(T)$ as well as related dynamic properties [1-6]. The linearized, derivativebased transformation of $\tau(T)$ data makes it possible to identify the range of its validity, namely [41],

$$
\begin{aligned}
& {\left[\frac{\mathrm{d} \ln \tau}{\mathrm{d}(1 / T)}\right]^{-1 / 2}=\left[\frac{H_{\mathrm{a}}(T)}{R}\right]^{-1 / 2}=\left(H_{\mathrm{a}}^{\prime}\right)^{-1 / 2}} \\
& =\left[\left(D_{T} T_{0}\right)^{-1 / 2}\right]-\frac{\left[T_{0}\left(D_{T} T_{0}\right)^{-1 / 2}\right]}{T}=A-\frac{B}{T}
\end{aligned}
$$

where $H_{\mathrm{a}}(T)$ denotes the apparent activation enthalpy and $R$ is the gas constant. 
A plot of $\left(H_{\mathrm{a}}^{\prime}\right)^{-1 / 2}$ versus $1 / T$ can yield optimal values of parameters, namely $T_{0}=B / A$ and $D_{T}=1 / A B$, and can identify the domain of validity of the VFT equation (1) prior to the final fitting of $\tau(T)$ data. In fact, equation (9) recalls the so-called 'Stickel plot', $\mathrm{d} \log _{10} \tau / \mathrm{d}(1 / T)$ versus $1 / T[87,88]$, introduced to estimate the dynamic crossover temperature $\left(T_{\mathrm{B}}\right)$ between 'dynamical domains', i.e. temperature regions described with different sets of parameters in the VFT relation. The universality of the timescale at the dynamic crossover $\tau\left(T_{\mathrm{B}}, P_{B}\right)=10^{-7 \pm 1} \mathrm{~s}$ is empirically supported [89].

A similar analysis can also support the PVFT (equation (5)) fit of $\tau(P)$ data, namely [41],

$$
\begin{aligned}
& {\left[\frac{\mathrm{d} \ln \tau}{\mathrm{d} P}\right]^{-1 / 2}=\left[\frac{V_{\mathrm{a}}}{R}\right]^{-1 / 2}=\left[V_{\mathrm{a}}^{\prime}\right]^{-1 / 2}} \\
& =\left(D_{P} P_{0}\right)^{-1 / 2} P_{0}-\left(D_{P} P_{0}\right)^{-1 / 2} P=A-B P
\end{aligned}
$$

where $V_{\mathrm{a}}$ is the apparent activation volume.

A plot of $\left(V_{\mathrm{a}}^{\prime}\right)^{-1 / 2}$ versus $P$ can show the range of validity of equation (5) and yield optimal values of the coefficients $P_{0}=A / B$ and $D_{P}=1 / A B$ prior to the final fitting of $\tau(P)$ data [41-43]. For plots employing equations (9) and (10) the non-sloping lines indicate the Arrhenius behavior [41].

There is a significant difference between the PVFT equation (5) and the VFT equation (1). For the latter the prefactor $\tau_{0}^{T}$ ranges from $\sim 10^{-12} \mathrm{~s}$ for molecular liquids ([1, 2, 4, 6, 41] and references therein) to $\sim 10^{-16} \mathrm{~s}$ for vitrifying, orientationally disordered crystals [42, 43]. Generally, the thermodynamic domain of the liquid state is limited by a spinodal, defining the stability limits for homogeneous nucleation [75-81]. Hence, one may expect the $\tau_{0}^{T}$ prefactor to be related to the relaxation time at the high temperature liquid-gas stability limit. However, for the PVFT equation (5) the prefactor $\tau_{0}^{P}$ is linked to the relaxation time at the atmospheric pressure, or any pressure $P>0.1 \mathrm{MPa}$ at which measurements started for a given isotherm. Consequently, it can take on arbitrary values ranging from picoseconds to even seconds [4, 5, 7, 9-14, 16-46].

We suggest that prefactors for the isobaric VFT equation (1) and a pressure counterpart should be linked to the appropriate stability limit (spinodal) and hence approach a similar timescale, namely $\tau_{0}, \tau_{0}^{P} \sim 10^{-12}$ s for low molecular weight liquids. This condition can be attained with a modified pressure counterpart of the VFT relation $[49,71]$ :

$$
\begin{gathered}
\tau(P)=\tau_{0}^{P} \exp \left[\frac{D_{P} \Delta P}{P_{0}-P}\right]=\tau_{0}^{P} \exp \left[\frac{D_{P} P-D_{P} P_{\mathrm{SL}}}{P_{0}-P}\right], \\
T=\mathrm{const}
\end{gathered}
$$

where $\Delta P=P-P_{\mathrm{SL}}$, and the prefactor $\tau_{0}^{P}$ is associated with the relaxation time at the liquid-gas stability limit at $P=P_{\mathrm{SL}}<0$.

Also for this equation, a derivative-based plot of $\left(V_{\mathrm{a}}^{\prime}\right)^{-1 / 2}$ versus $P$ yields a linear dependence, namely $[49,71]$,

$$
\begin{gathered}
{\left[\frac{\mathrm{d} \ln \tau}{\mathrm{d} P}\right]=\left[V_{\mathrm{a}}^{\prime}\right]^{-1 / 2}=\left[D_{P}\left(P_{0}-P_{\mathrm{SL}}\right)\right]^{-1 / 2} P_{0}} \\
-\left[D_{P}\left(P_{0}-P_{\mathrm{SL}}\right)\right]^{-1 / 2} P=A+B P .
\end{gathered}
$$

In this case, $P_{0}=A / B$ as for equation (10), but $1 / A B=D_{P} P_{0} /\left(P_{0}-P_{\mathrm{SL}}\right)$. The comparison of the 'new'
PVFT equations (11) and the 'old' PVFT equation (5) shows that the latter is associated with unphysical loci of the gasliquid stability limit at $P_{\mathrm{SL}}(T)=0$ for arbitrary temperature. This artifact leads to significantly different values of fragility strength coefficients for these expressions, namely,

$$
\frac{D_{P}^{\text {'old' }}}{D_{P}^{\text {'new' }}}=\frac{P_{0}}{P_{0}-P_{\mathrm{SL}}}
$$

Practical possibilities for equation (11) for portraying $\tau(P)$ data were recently shown for glycerol [49] and epoxy resin EPON 828 [71]. It is worth stressing that the modified PVFT equation (11) seems to be more self-consistent than the basic VFT equation (1). First, the $\tau_{0}^{P}$ prefactor is clearly linked to the stability limit ('homogeneous breaking') loci at $P=P_{\mathrm{SL}}$. Second, equation (11) can be smoothly transformed into an Arrhenius-type pressure dependence.

The novel PVFT equation (11) offers a possibility hardly, if at all, formulated so far, namely, the extrapolation of $\tau(P)$ evolution into the negative pressure domain. The significance of the negative pressures concept for resolving the glass transition puzzle has been pointed out several times [1, 49, 71, 75-81]. It is noteworthy that for many physical properties of liquids there is clear evidence for continuous evolution from the stable hydrostatic ('positive') pressures region to the inherently metastable negative pressures $(P<0)$ domain. There are no hallmarks of passing through $P=0([75,76,80,90,91]$ and references therein). Unfortunately, determining the loci of the stability limit (spinodal) as well as making direct measurements in the negative pressures domain remain extremely challenging experimental problems [76].

\section{The Avramov-model-based description}

When portraying $\tau(T), \tau(P)$ or $\eta(T), \eta(P)$ experimental data with a given relation the question of the domain of its validity always arises. Its inappropriate estimation can lead to erroneous values of parameters in fitting. For instance, this may occur if a single VFT equation is used to describe experimental data also beyond the given dynamical domain. However, for VFT and PVFT equations the derivative-based analysis, discussed above, can show the domains of their validity and yield optimal values of relevant parameters prior to the final fitting of $\tau(T)$ or $\tau(P)$ data. A similar distortion-sensitive analysis can be proposed for the AvM-type equations (6) and (7), namely,

$$
\begin{aligned}
& \log \left[\frac{\mathrm{d}(\ln \tau)}{\mathrm{d}(1 / T)}\right]=\log H_{\mathrm{a}}^{\prime}=\log (C D)+(1-D) \log T, \\
& \quad \text { for } P=\mathrm{const}
\end{aligned}
$$

where $C=\varepsilon\left(T_{\mathrm{g}}\right)^{D}$ and $D=\alpha$ for the Avramov model.

The AvM equation (7) offers the possibility of describing both the temperature and pressure dependences of dynamic properties. Originally, the pressure behavior in equation (7) was introduced via the $T_{\mathrm{g}}(P)$ dependence in the form of the AA equation (8) [68, 69]. However, this expression cannot be extended into the negative pressures region. Additionally, 
for the AA equation (8) values of $T_{\mathrm{g}}$ can only increase on compressing whereas under extreme pressures the asymptotic behavior or a maximum of $T_{\mathrm{g}}(P)$ is anticipated ([1, 49, 71] and references therein). It also seems that the ultimate relation for describing $T_{\mathrm{g}}(P)$ behavior should exhibit a negative pressure asymptote ([1] and references therein). It is noteworthy that the reference temperature $T_{\mathrm{g}}^{0}$ in the AA equation (8) is related to $P=0$, but in practice it is linked to the atmospheric pressure value, to reduce the number of fitted parameters. This has some importance for researchers on our planet, Earth. However, for hypothetical research on Jupiter or Saturn such estimation of $T_{\mathrm{g}}^{0}$ would lead to significant distortions when using equation (8). In fact, all of these comments are valid also for the pressure evolution of the melting temperature described via the basic Simon-Glatzel equation ([71] and references therein). These parasitic artifacts are absent for the recently proposed extension of equation (8), namely [49, 71],

$T_{\mathrm{g}}(P)=F(P) D(P)=T_{\mathrm{g}}^{0}\left(1+\frac{\Delta P}{\pi+P_{\mathrm{g}}^{0}}\right)^{1 / b} \exp \left(-\frac{\Delta P}{c}\right)$

where $F(P)$ is the rising function and $D(P)$ the damping function; $\Delta P=P-P_{\mathrm{g}}^{0} ; P_{\mathrm{g}}^{0}$ and $T_{\mathrm{g}}^{0}$ are the reference pressure $\left(P_{\text {ref }}\right)$ and temperature $\left(T_{\text {ref }}\right)$ from which the $T_{\mathrm{g}}(P)$ estimation begins; $c$ denotes the damping pressure coefficient; $-\pi$ is the negative pressure asymptote for $T \rightarrow 0$; the coefficient $\Pi=\pi+P_{\mathrm{g}}^{0}$.

For moderate pressures, $T_{\mathrm{g}}(P)$ is governed solely by the $F(P)$ term, whereby [49]

$$
\left[\frac{\mathrm{d}\left(\ln T_{\mathrm{g}}\right)}{\mathrm{d} P}\right]^{-1}=b \pi+b P .
$$

The application of equation (16) can indicate the domain of validity of equation (15) as well as the possible significance of the damping term. It also shows that equation (15) is governed by the pressure-invariant coefficients $\pi$ and $b$, estimated via a linear regression fit. On the basis of equations (15) and (16), a form of the AvM relations with pressure-invariant coefficients can be proposed, namely,

$$
\begin{aligned}
& \ln \tau_{\text {scaled }}=\ln \left[\frac{\tau(P)}{\tau_{0}}\right] \\
& =\frac{\varepsilon}{T_{\text {ref }}^{D}}\left[T_{\text {ref }}\left(1+\frac{\Delta P}{\pi+P_{\text {ref }}}\right)^{1 / b}\right]^{D} .
\end{aligned}
$$

The modified AvM dependence can be extended into the negative pressures domain, in contrast to the 'basic' equation (7). To identify the range of its validity and to estimate optimal values of parameters, again a derivative-based analysis can be proposed:

$$
\begin{gathered}
{\left[\frac{\mathrm{d}\left[\ln \left(\ln \tau_{\text {scaled }}\right)\right]}{\mathrm{d} P}\right]^{-1}=D^{-1}\left[\frac{\mathrm{d} \ln T_{\mathrm{g}}}{\mathrm{d} P}\right]^{-1}} \\
=D^{-1}(\pi b+b P)=A+B P .
\end{gathered}
$$

For glass-forming materials or for extreme ranges of pressures, the damping term $(D(P))$ in equation (15) may be significant.
In such a case, the following transformation of experimental data for the modified AvM expression can be proposed:

$$
\left[\frac{\mathrm{d}\left[\ln \left(\ln \tau_{\text {scaled }}\right)\right]}{\mathrm{d} P}+D c^{-1}\right]^{-1}=D^{-1}(\pi b+b P)=A+B P .
$$

For the optimal selection of the damping coefficient $c$ the plot based on equation (19) should yield a linear dependence. Subsequently, optimal values of $b$ and $\pi$ coefficients can be estimated via the subsequent linear regression fit.

\section{The comparison of VFT- and AvM-based descriptions}

In this section temperature and pressure dependences of structural (dielectric) relaxation times $(\tau(T)$ and $\tau(P))$ for diethyl phthalate (DEP) and propylene carbonate (PC) are tested using VFT, PVFT and AvM relations, supported by the derivative-based analysis. It is worth recalling that pressure measurements of the dielectric relaxation time are limited to frequencies $f<10 \mathrm{MHz}$ [4, 5, 7-50], due to still existing technical restrictions. Hence, usually $\tau(P)$ experimental data cannot cover the timescale $\tau<\tau\left(P_{B}\right) \sim 10^{-7} \mathrm{~s}[28,75]$. However, for DEP and PC it was possible to estimate $\tau(P)$ behavior both above and below the crossover pressure $\left(P_{B}\right)$, due to the scaling of $\tau(T)$ and $\sigma(T)$ data. These estimations were based on data from [23, 41] for DEP and [29] for PC. The analysis presented also employs $T_{\mathrm{g}}(P)$ dependences, recalling data from [23] for DEP and [39] for PC. The latter data have to be supplemented with the authors' measurements to enable a reliable derivative-based analysis to be made. The experimental set-up, based on the BDS 80 Novocontrol impedance analyzer, is described in [5, 49]. The glass transition was determined by reaching the temperature at which $\tau\left(T_{\mathrm{g}}, P_{\mathrm{g}}\right)=100 \mathrm{~s}$, where $\tau=1 / 2 \pi f_{\mathrm{P}}$ and $f_{\mathrm{P}}$ is the peak frequency of the dielectric loss curve. Values of $T_{\mathrm{g}}(P)$ for $P=$ $0.14,0.29,0.41,0.41,0.72 \mathrm{GPa}$ for DEP and for $P=0.6$, 0.7 and $0.8 \mathrm{GPa}$ for PC were determined. Values of all fitted parameters are always given in figures presented below.

Figure 1 shows $\tau(T)$ data for DEP portrayed using the VFT equation (1) and the AvM-type equation (7). The VFT parameterization invokes results of the derivative-based analysis (equation (9)) from [41]. This parameterization led to two sets of $\tau_{0}^{T}, D_{T}$ and $T_{0}$, associated with subsequent dynamical domains and the crossover at $\tau_{\mathrm{B}}\left(T_{\mathrm{B}} \approx 220 \mathrm{~K}\right) \approx$ $0.7 \mu \mathrm{s}$. It is noteworthy that using the condition $\tau\left(T_{\mathrm{g}}\right)=100 \mathrm{~s}$ one can estimate the 'real' glass transition just on the basis of the low temperature dynamical domain $\left(T_{\mathrm{g}}<T<T_{\mathrm{B}}\right)$. For the high temperature domain $\left(T>T_{\mathrm{B}}\right)$ the virtual glass $\left(T_{\mathrm{g}}\right)$ and 'VFT ideal glass' $\left(T_{0}\right)$ temperatures appear.

The inset in figure 1 present results of the derivative-based analysis (equation (18)) focusing on the AvM-type description (equations (6) and (7)). The parameterization obtained in this way is shown by the dotted curve in the main part of figure 1. The lack of dynamic crossover for the AvM-type description is noteworthy.

Figure 2 presents results of VFT (equations (1)) and AvM (equations (6) and (7)) parameterizations of $\tau(T)$ data 


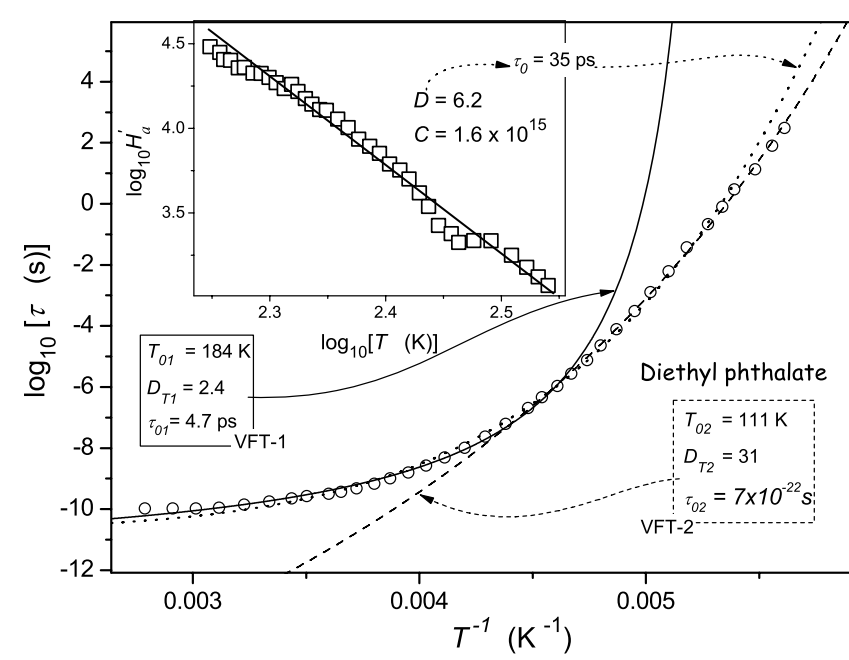

Figure 1. The Arrhenius plot of the structural relaxation times for diethyl phthalate $[23,41]$ under atmospheric pressure. The solid and the dashed curves are for the VFT description (equation (1)) in subsequent dynamical domains $T>T_{\mathrm{B}}$ and $T<T_{\mathrm{B}}$, respectively. Values $D_{T}$ and $T_{0}$ for these domains were obtained via the linearized, derivative-based analysis (equation (9)) [41]. The dotted curve is linked to AvM equations (6) and (7). Optimal values of $C$ and $D$ parameters were estimated via the derivative-based analysis (equations (14)), whose results are shown in the inset.

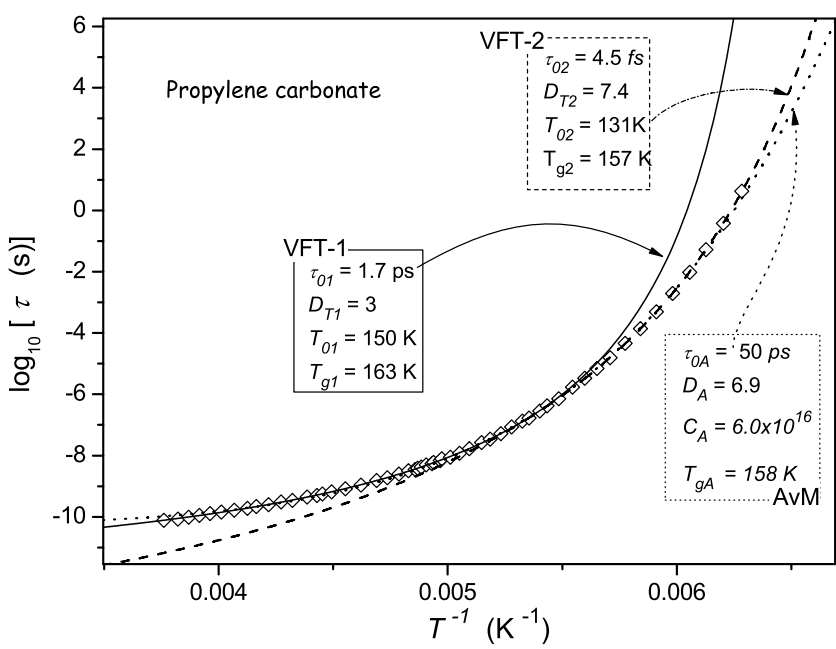

Figure 2. The Arrhenius plot of the structural relaxation times for propylene carbonate [29] under atmospheric pressure. The solid and the dashed curves are for the VFT description (equation (1)) in subsequent dynamical domains $T>T_{\mathrm{B}}$ and $T<T_{\mathrm{B}}$, respectively. Values $D_{T}$ and $T_{0}$ for these domains were obtained via the linearized, derivative-based analysis (equation (9)), as shown in figure 3 . The dotted curve is linked to equation (6) and the equivalent AvM equation (7). Optimal values of $C$ and $D$ parameters were estimated via the derivative-based analysis (equation (14)), whose results are given in figure 3

for PC. They are based on the derivative-based analyses whose results are given in figure 3 . For the VFT description also, two dynamical domains are visible, with the dynamical crossover at $\tau_{\mathrm{B}}\left(T_{\mathrm{B}} \approx 195 \mathrm{~K}\right) \approx 0.4 \times 10^{-7} \mathrm{~s}$. Both for $\mathrm{DEP}$ and for $\mathrm{PC}$ the value of $D_{T}$ is significantly larger for the dynamical domain $T_{\mathrm{g}}<T<T_{\mathrm{B}}$ than for the high

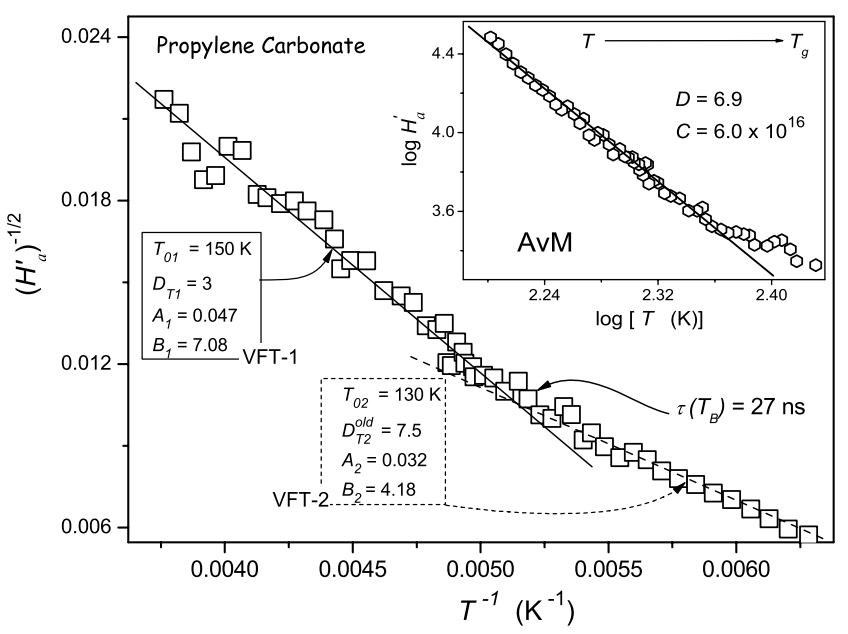

Figure 3. The derivative-based analysis of $\tau(T)$ data for propylene carbonate from figure 2 , focusing on the validity of the VFT description (equation (9)). A similar analysis linked to the AvM type via equation (14) is shown in the inset. Values of parameters, relevant for the final fits via equations (1) and (6), are given in the figure. $H_{\mathrm{a}}^{\prime}=\mathrm{d} \ln \tau / \mathrm{d}(1 / T)$ is related to the apparent activation enthalpy.

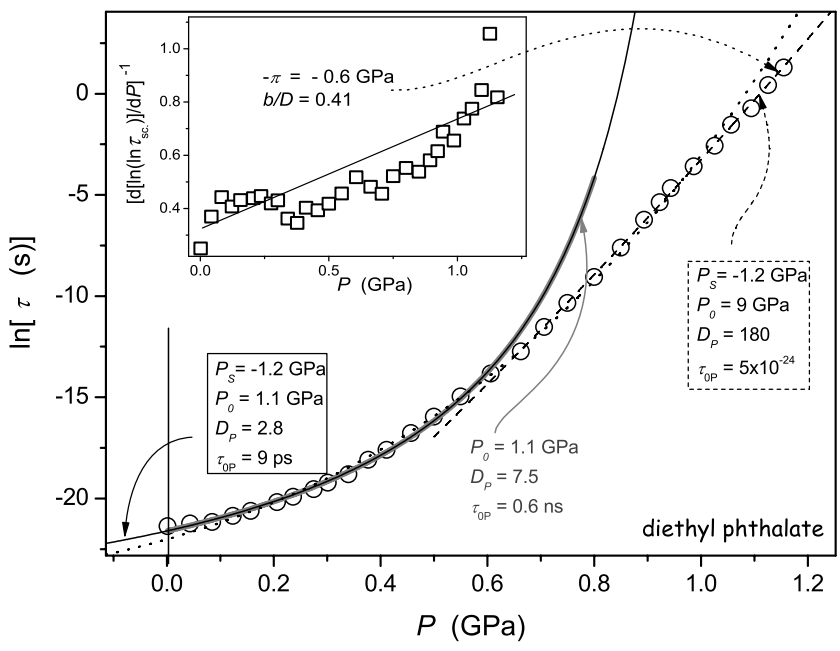

Figure 4. The Arrhenius plot for the isothermal ( $T=293 \mathrm{~K}$ ) pressure evolution of structural relaxation times for diethyl phthalate [23, 41]. The solid, thick, gray curve shows the 'old' PVFT equation (5). The solid 'thin' and the dashed curves are for the 'new' PVFT equation (11) in following dynamical domains. Values of $D_{P}$ and $P_{0}$ were estimated via the derivative-based plot $\left(V_{\mathrm{a}}^{\prime}\right)^{-1 / 2}$ versus $P$ (see equations (10) and (12)). The dotted curve is related to the AvM-type equation (17). Values of parameters $C$ and $D$ were estimated using the derivative-based analysis introduced by equation (18). Their results are shown in the inset.

temperature domain $T>T_{\mathrm{B}}$. The inset in figure 3 shows results of the derivative-based analysis related to the AvM-type parameterization (equation (18)).

On the basis of optimal values of $D$ and $C$ coefficients obtained in the inset in figure 3 , one can reduce the fitting of $\tau(T)$ data via equations (6) or (7) in figure 2 solely to that of the prefactor. The resulting curve can portray the whole set of tested experimental data, although some distortions appear.

Figure 4 shows the Arrhenius plot for the isothermal, pressure dependence of the primary relaxation time in DEP. 


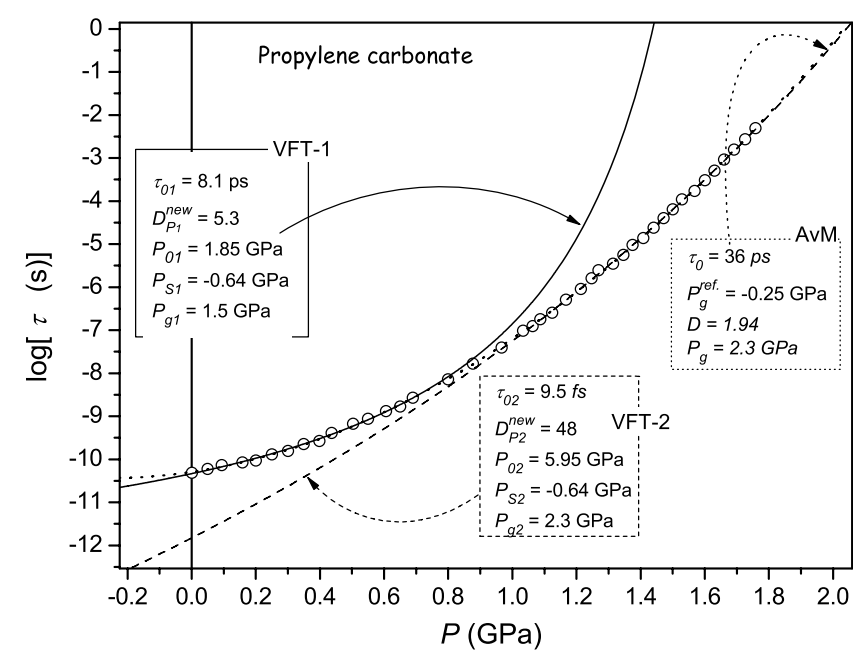

Figure 5. The Arrhenius plot for the isothermal ( $T \approx 273 \mathrm{~K}$ ) pressure evolution of structural relaxation times for propylene carbonate [29]. The solid and the dotted curves are for the 'new' PVFT equation (8), valid within dynamical domains $P<P_{B}$ and $P>P_{B}$. Values of $P_{0}, D_{P}$ and $P_{S}$ were supported by the derivative-based analysis given in figure 6 . The dotted curve is associated with the AvM equation (17), linked to the modified SG-type equation (15) for $T_{\mathrm{g}}(P)$ evolution.

The derivative-based analysis, associated with equations (10) and (12), yielded the following values of coefficients [34]: $A=0.39$ and $B=0.35$ (for $\tau$ (s) and $P(\mathrm{GPa})$ ) below the dynamic crossover pressure $P_{B}$. For $P>P_{B}$ the Arrhenius dependence was suggested in [34]. However, our revised derivative-based analysis indicated the possibility of a 'very strong-type' VFT behavior in this domain. Results of parameterizations employing these results and based on the 'new' PVFT equation (11) are shown by the solid and the dashed curves in figure 4 , respectively, for subsequent dynamical domains. The 'thick' gray curve recalls results from [41]: it is linked to the 'old' PVFT equation (5). The difference in values of the fragility strength coefficients when using equations (5) and (11) is noteworthy: $D_{P}^{\text {old }}=7.5$ and $D_{P}^{\text {new }}=2.8$. Only equation (11) enables an extension of $\tau(P)$ evolution into the negative pressures domain to be made.

The inset in figure 4 shows results of the derivativebased analysis focused on the AvM-type equation (17). The parameters obtained in this way can be used to portray $\tau(P)$ dependence for the entire range of pressures (figure 4), without any hallmark when passing the dynamic crossover, clearly manifested for the PVFT description.

Figure 5 presents the pressure behavior of dielectric relaxation times for propylene carbonate, for a selected isotherm. The parameterization of experimental data is supported by values of parameters obtained using linearized, derivative-based transformation of $\tau(P)$ whose results are given in figure 6, both for the PVFT (equations (11) and (12)) and for the AvM-type (equations (17) and (18)) descriptions.

The behavior obtained is analogous to the one discussed above for DEP. Also in this case the parameterization using the modified PVFT (equation (11)) and AvM (equation (17)) expressions can be extended into the negative pressures domain.

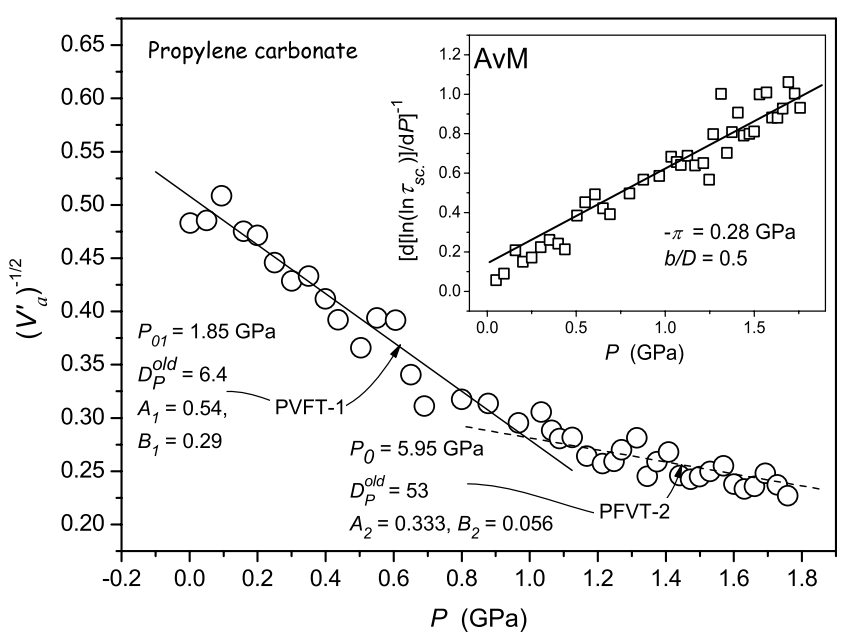

Figure 6. The linearized, derivative-based analysis of $\tau(P)$ data for propylene carbonate (figure 5) focused on the validity of PVFT equations (5) and (11). A similar analysis focused on the AvM equation (7) supplemented with the modified SG-type equation (15) is shown in the inset. Values of parameters obtained by the linear regression fit, via equations (10), (12) and (18), are given in the figure. $V_{\mathrm{a}}^{\prime}=\mathrm{d} \ln \tau(P) / \mathrm{d} P$ is related to the apparent activation volume.

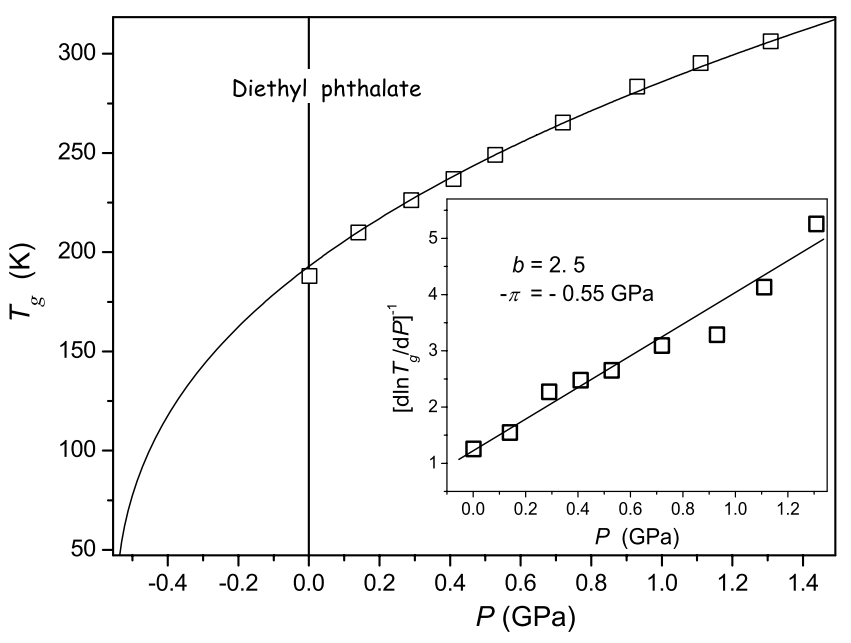

Figure 7. Pressure dependence of the glass temperature for diethyl phthalate ([23] supplemented with the authors' measurements) portrayed by the modified SG-type equation (15). Values of all relevant parameters, namely the negative pressure asymptote $\pi$ and the power exponent $b$, were estimated via the derivative-based analysis shown in the inset; equation (16).

Figures 7 and 8 present the pressure evolution of the glass temperature for DEP and for PC, respectively. The solid curves in figures 7 and 8 show the description via the modified SGtype equation (15). For both glass formers the estimates of $\pi$ and $b$ values were taken from the supplementary derivativebased analysis via equation (16). This is shown in the inset in figure 7 . We noted the negligible influence of the damping term for the range of pressures tested. Such behavior agrees with results obtained for di-isooctyl phthalate, for which the $D(P)$ term in equation (15) was significant only for $P>$ $15 \mathrm{GPa}[41]$. 


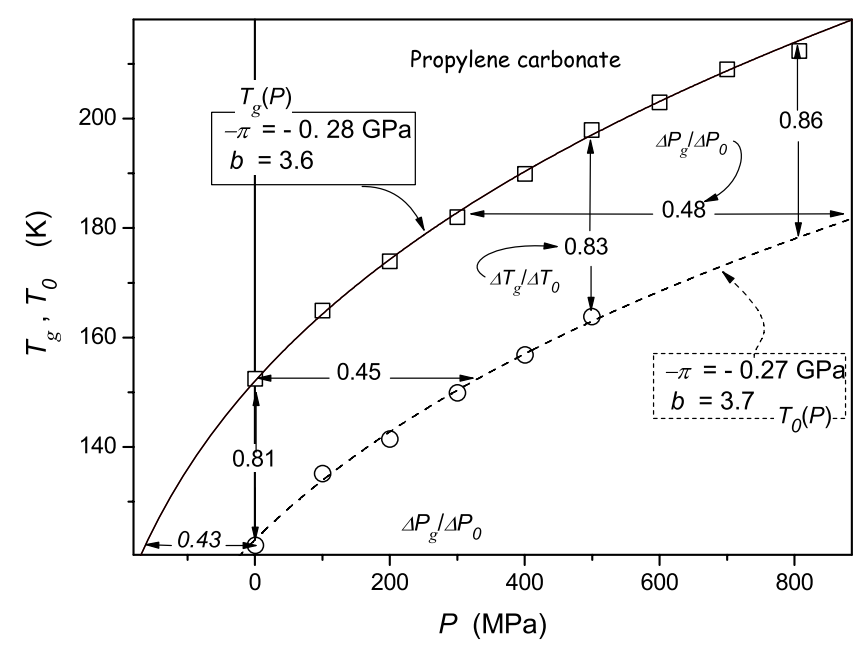

Figure 8. Pressure dependence of the glass temperature $T_{\mathrm{g}}(P)$ and the VFT-based estimation of the ideal glass temperature $T_{0}(P)$ for propylene carbonate, based on data from [39] supplemented with the authors' measurements. The solid curve is related to the modified SG-type equation (15). Values of $T_{\mathrm{g}}(P)$ for $P \geqslant 600 \mathrm{MPa}$ were determined by the authors via reaching the $\tau\left(T_{\mathrm{g}}, P_{\mathrm{g}}\right)=100 \mathrm{~s}$ condition in BDS measurements. The solid vertical arrows show $T_{0} / T_{\mathrm{g}}$ values on pressurizing. The dashed horizontal arrows indicate values of $\Delta P_{\mathrm{g}} / \Delta P_{0}$ for the following isotherms, taking the values of the negative pressure asymptotes $(\pi)$ given in the figures as the reference.

The derivative analysis yielded: (i) $-\pi=-0.55 \mathrm{GPa}$ and $b=2.5$ for DEP and (ii) $-\pi \approx-0.3 \mathrm{GPa}$ and $b=3.6$ for PC.

These results are in fair agreement with values estimated via the AvM-based equation (17): (i) $-\pi=-0.6 \mathrm{GPa}$ and $b / D=-0.41$ and hence $b=2.5$ for $D=6.2$ (DEP: figures 1 and 4 ), (ii) $-\pi=-0.28 \mathrm{GPa}$ and $b / D=-0.5$ which yields $b=3.5$ for $D=6.9$ (PC: figures 2 and 5).

For PC the evolution of both $T_{\mathrm{g}}(P)$ and $T_{0}(P)$ is shown in figure 8. The changes of the ratios $T_{\mathrm{g}} / T_{0}$ and $\Delta P_{\mathrm{g}} / \Delta P_{0}$ on pressurizing are noteworthy. On the basis of the SG-type equation (15) it was possible to estimate their values, even taking $P=0$ as the reference.

Direct determination of $T_{\mathrm{g}}(P)$ dependence is a challenging experimental task associated with significant experimental problems, particularly when covering extreme pressure domains. However, results presented above show that the estimation of $T_{\mathrm{g}}(P)$ evolution is possible just on the basis of a single set of $\tau(T)$ and $\tau(P)$. Moreover, $T_{\mathrm{g}}(P)$ behavior predicted in this way may be valid even well above the range of pressures used in $\tau(P)$ measurements, due to the pressure-invariant character of key parameters in the SG-type equation (15). One may expect such analysis to be applicable also for $\eta(T)$ and $\eta(P)$ or $\sigma(T)$ and $\sigma(P)$ sets of data. We would like to stress the possible significance of $T_{\mathrm{g}}(P)$ estimations as regards both fundamental and technological issues. The latter can be related to the pressure induced amorphization (PIA) [92] or applications in the pharmaceutical industry [93, 94].

\section{Pressure dependence of fragility}

A metric for the evolution of the structural relaxation time or viscosity is called fragility. It was introduced via the so-called
'Angell plot' [82, 83], $\log _{10} \eta$ or $\log _{10} \tau$ versus $T_{\mathrm{g}} / T$, enabling a common, 'universal' presentation of 'dynamic' data for different glass-forming materials [1, 6, 82, 83]. For such plots, so-called 'strong' liquids exhibit an almost linear, Arrheniuslike, behavior. 'Fragile' liquids exhibit a strong nonlinearity of the plot, showing clearly non-Arrhenius evolution of viscosity or relaxation times. The fragility can be quantified by the steepness index $(m)$ which is just the slope in the Angell plot evaluated at $T_{\mathrm{g}}[1,82,83]$. The relationship between $m$ and $D_{T}$ dates to the paper of Böhmer et al [83], who noted that $m=$ $m_{\min }+m_{\min }^{2} / D_{T}=16+590 / D_{T}\left(m_{\min }=\log _{10} \tau\left(T_{\mathrm{g}}\right) / \tau_{0}^{T}\right.$, assuming $\tau_{0}^{T}=10^{-14} \mathrm{~s}$ and $\tau\left(T_{\mathrm{g}}\right)=100 \mathrm{~s}$ ). For $m>80$ and $D_{T}<10$, liquids are considered fragile, whereas $m<30$ and $D_{T}>10$ give a description of strong glass formers. All of this can suggest the equivalence of $m$ and $D_{T}$ as metrics of the fragility $[1-6,73,83]$. It is noteworthy that the fragility is considered one of key characteristics of glassy materials. Several other metrics for fragility were also proposed [1, 6, 73].

One of the most important problems associated with the concept of fragility is the question 'Does fragility depend on pressure?' from the title of [13], in which consequences of the aforementioned definition of the fragility for the description of $\tau(T)$ and $\tau(P)$ data via the VFT equation (1) and the PVFT equation (5) were considered, namely,

$$
\begin{gathered}
m_{P}\left(T \rightarrow T_{\mathrm{g}}\right)=\left[\frac{\partial \log \tau}{\partial\left(T_{\mathrm{g}} / T\right)}\right]_{T \rightarrow T_{\mathrm{g}}}^{P=\mathrm{const}} \\
=\frac{1}{\log _{10}(e)} \frac{D_{T}\left(T_{0} / T_{\mathrm{g}}\right)}{\left(1-T_{0} / T_{\mathrm{g}}\right)^{2}}=A+\frac{A^{\prime 2}}{D_{T}}, \\
m_{T}\left(P \rightarrow P_{\mathrm{g}}\right)=\left[\frac{\partial \log \tau}{\partial\left(P / P_{\mathrm{g}}\right)}\right]_{P \rightarrow P_{\mathrm{g}}}^{T=\text { const }} \\
=\frac{1}{\log _{10}(e)} \frac{D_{P}\left(P_{\mathrm{g}} / P_{0}\right)}{\left(1-P_{\mathrm{g}} / P_{0}\right)^{2}}=B+\frac{B^{\prime 2}}{D_{P}},
\end{gathered}
$$

where $A=\log \tau\left(T_{\mathrm{g}}\right)-\log \tau_{0}^{T}=2-\log \tau_{0}^{T}$ and $B=$ $\log \tau\left(T_{\mathrm{g}}\right)-\log \tau_{0}^{P}=2-\log \tau_{0}^{P}$, with $\tau\left(T_{\mathrm{g}}, P_{\mathrm{g}}\right)=100 \mathrm{~s}$, $A^{\prime}=A / \ln 10$, and $B^{\prime}=B / \ln 10$. For tests under atmospheric pressure, $m_{P}\left(T \rightarrow T_{\mathrm{g}}\right)=m$.

Equations (20) and (21) were obtained using the VFT equation (1) and the PVFT equation (5), respectively.

It was concluded in [13] that: (i) ' $m_{P}$ and $D_{T}$ are equivalent measures of fragility if $A$ is pressure invariant, and thus relaxation times for different isotherms will fall on a single master curve when plotted versus $T_{\mathrm{g}} / T$ ' and (ii) 'since the preexponential factor in equation (5) (which is just the ambient pressure value of $\tau$ ) varies with temperature, $m_{P}$ will always decrease with decreasing temperature, so that isotherms of $\tau(P)$ will not collapse onto a single master curve.'

Probably the fundamental the non-equivalence of $m_{P}$ and $m_{T}$ can be recognized as the key reason for the very limited application of the isothermal, pressure-related fragility $m_{T}$ in the last few years.

As regards the isobaric fragility $m_{P}$ (equation (20)), the comments recalled from [13] can be associated with changes of $T_{0} / T_{\mathrm{g}}$ on pressurizing (equation (20)), particularly assuming $D_{T} \approx$ const. The analysis of the behavior of $m_{P}$, employing 
$T_{\mathrm{g}}(P)$ and $T_{0}(P)$ parameterizations via equation (15), is possible for propylene carbonate (figure 8) and DGEBA (EPON 828; see the results given in [71]).

As regards DGEBA, the value of $T_{0} / T_{\mathrm{g}}$ changes from (a) 0.89 at $P=0.1 \mathrm{MPa}$ to (b) 0.875 at $P=2 \mathrm{GPa}$ [71], which yields $m_{P}^{a} / m_{P}^{b} \approx 1.1$ according to equation (20). For PC, from results given in figure 8 one can estimate: (a) $T_{0} / T_{\mathrm{g}} \approx 0.81$ at $P=0.1 \mathrm{MPa}$ and $(\mathrm{b}) T_{0} / T_{\mathrm{g}} \approx 0.82_{3}$ at $P=0.5 \mathrm{GPa}$, which yields $m_{P}^{(a)} / m_{P}^{(b)} \approx 0.9$. Then, a weak pressure dependence of the temperature-related, isobaric fragility appears. However, the last term in equation (20) can suggest the lack of a pressure dependence of $m_{P}$, if the assumption $A=m_{\min }=\log _{10} \tau_{0}^{T}=10^{-14} \mathrm{~s}$ for $T \gg$ $T_{\mathrm{g}}$ is recalled [13, 83]. In the opinion of the authors the universality of $m_{\min }$, linked to the $\tau_{0}^{T}=10^{-14} \mathrm{~s}$ prefactor, may be questioned. First, different prefactors are obtained in VFT/PVFT equations for the dynamical domains close to and remote from the glass transition $\left(T_{\mathrm{B}}, P_{B}\right)$ [41]. Second, the analysis of available experimental data seems to indicate that for $T_{\mathrm{g}} / T \rightarrow 0$ prefactors can change from $\sim 10^{-11} \mathrm{~s}$ for some molecular fragile glass formers $[41,71]$ to $\sim 10^{-16} \mathrm{~s}$ for plastic crystals $[42,43]$. We wish to indicate that the pressure dependence of $\tau_{0}^{T}$ may appear if this prefactor is linked to the high temperature liquid-gas stability limit. It is noteworthy that the pressure independence of the fragility $m_{P}$ was recently suggested in [39], recalling the defect diffusion model [54] and assuming the hypothetical pressure independence of the $T_{\mathrm{g}} / T_{\mathrm{m}}$ ratio. In this respect the clear decrease of the $T_{\mathrm{g}} / T_{\mathrm{m}}$ ratio on pressurizing in selenium is noteworthy: from about 0.67 to 0.5 , reported in [71] recently.

For the isothermic, pressure-related, fragility $m_{T}$ (equation (21)) a similar analysis can lead to confusing results, particularly when taking the atmospheric pressure $P=0.1 \mathrm{MPa}$ as the reference. This is possible when portraying $T_{\mathrm{g}}(P)$ and $T_{0}(P)$ behavior via the modified SG-type equation (15), which includes the negative pressure domain.

For PC one can estimate (figure 8):

(a) $P_{\mathrm{g}}^{(a)} / P_{0}^{(a)} \approx 0.1 \mathrm{MPa} / 160 \mathrm{MPa} \approx 3 \times 10^{-4}$ for $T=$ $T_{\mathrm{g}}(P=0.1 \mathrm{MPa})$.

(b) $P_{\mathrm{g}}^{(b)} / P_{0}^{(b)} \approx 300 \mathrm{MPa} / 903 \mathrm{MPa} \approx 0.33$ for $T=$ $T_{\mathrm{g}}(P=0.3 \mathrm{GPa})$.

Consequently, equation (21) yields $m_{T}^{(a)} / m_{T}^{(b)} \approx 1 / 100($ !) for $D_{P} \approx$ const.

For DGEBA one can conclude from results shown in [71]:

(a) $P_{\mathrm{g}}^{(a)} / P_{0}^{(a)}=0.1 \mathrm{MPa} / 2.27 \mathrm{GPa} \approx 4.4 \times 10^{-5}$ for $T=$ $T_{\mathrm{g}}(0.1 \mathrm{MPa}) \approx 256 \mathrm{~K}$.

(b) $P_{\mathrm{g}}^{(b)} / P_{0}^{(b)}=2.7 \mathrm{GPa} / 7.9 \mathrm{GPa} \approx 0.34$ for $T \approx 292 \mathrm{~K}$.

These values give $m_{T}^{(a)} / m_{T}^{(b)} \approx 3 \times 10^{-3}(!)$.

Hence, both for PC and for DGEBA, the 'anomaly' in the evolution of $m_{T}$ for $P \rightarrow 0$ appears (!). This 'singularity' is the consequence of the middle term in equation (21). A discussion of the pressure or temperature dependence of $m_{T}$ via the last term in equation (21), $B=\log \tau\left(T_{\mathrm{g}}\right)-$ $\log \tau_{0}^{P}=2-\log \tau_{0}^{P}$, is meaningless due the arbitrariness of $\tau_{0}^{P}(T=$ const $)$ values, as mentioned above.
The non-physical pressure behavior of the isothermal fragility $m_{T}$ and the non-equivalence of $m_{T}$ and $m_{P}$ [13] may be considered as consequences of the ill-defined 'old' PVFT equation (5), because of the non-physical loci of the stability limit at $P_{\mathrm{SL}}=0$. To avoid these parasitic artifacts we propose to correct the definition of the isothermic, pressure-related steepness index and fragility, on the basis of the novel PVFT equation (11), namely,

$$
\begin{aligned}
m_{T} & =\left[\frac{\mathrm{d} \log _{10} \tau}{\mathrm{d} \Delta P / \Delta P_{\mathrm{g}}}\right]_{P \rightarrow P_{\mathrm{g}}}^{T=\text { const. }}=\frac{1}{\ln 10} \frac{D_{P}\left(\Delta P_{\mathrm{g}} / \Delta P_{0}\right)}{\left(1-\Delta P_{\mathrm{g}} / \Delta P_{0}\right)^{2}} \\
= & B+\frac{B^{\prime 2}}{D_{P}}
\end{aligned}
$$

where $\Delta P=P-P_{\mathrm{SL}}$ for the given isotherm, $\Delta P_{\mathrm{g}}=P_{\mathrm{g}}-\pi$, $\Delta P_{0}=P_{0}-\pi$

Its application yields $m_{T}^{(a)} / m_{T}^{(b)} \approx 0.89$ for $\mathrm{PC}$ and $m_{T}^{a} / m_{T}^{b} \approx 0.86$ for DGEBA, for the same isotherms as above. For the isothermic fragility defined by equation (22) there is no 'singularity' for $P \rightarrow 0$. The weak pressure dependence of $m_{T}$ along $T_{\mathrm{g}}(P)$ is seen to be consistent with the last term in equation (22) if the link of $\tau_{0}^{P}$ to the gas-liquid stability limit in equation (11) is recalled.

Hence, for the novel PVFT equation (11) and the modified isothermic fragility (equation (22)) the total equivalence of $m_{T}$ and $m_{P}$ and then the possibility of scaling $\tau(T)$ and $\tau(P)$ data appears. This resolves the puzzling disagreement between $m_{T}$ and $m_{P}$ stated in [13].

Please note that in first studies on the 'fragility under pressure' issue, the isothermic, pressure-related fragility was denoted as $m_{P}$ and the isobaric, temperature-related fragility as $m_{T}[10,13,14,16-23]$. Recently, the opposite symbols have been used, namely $m_{T}$ for the isothermic steepness index and fragility and $m_{P}$ for the isobaric steepness index (fragility). Equations (20) and (21) follow this notation. Unfortunately, a similar change can be noted also for fragility strength coefficients $D_{T}$ and $D_{P}$. For instance in [95] the symbol $D_{P}$ was linked to the VFT equation (1) and $D_{T}$ to the PVFT equation (5), contrary to the case for earlier studies. Hence, to avoid confusion the meaning of the given set of symbols related to the fragility should always be tested.

\section{A pressure counterpart of the 'Angell plot'}

The Angell plot is probably one of the most often invoked hallmarks of the glass transition [1-6, 73, 82, 83]. This plot also enables a comparison of the dynamics of different glassforming systems to be made, irrespective of their microscopic bases. Hence, in a natural way the question of a pressure counterpart of the Angell plot arises. Surprisingly, there seems to be no consistent proposal for such a plot given so far. The simple parallel of the basic Angell plot, $\ln \eta$ or $\ln \tau$ versus $P / P_{\mathrm{g}}$ [96], cannot be considered as a real pressure counterpart, for reasons indicated in the preceding section when discussing the non-equivalence of $m_{T}$ and $m_{P}$ steepness indexes (fragilities).

Figure 9 presents a novel proposal for a pressure counterpart of the Angell plot which is based on the modified PVFT equation (11) and the definition of the 


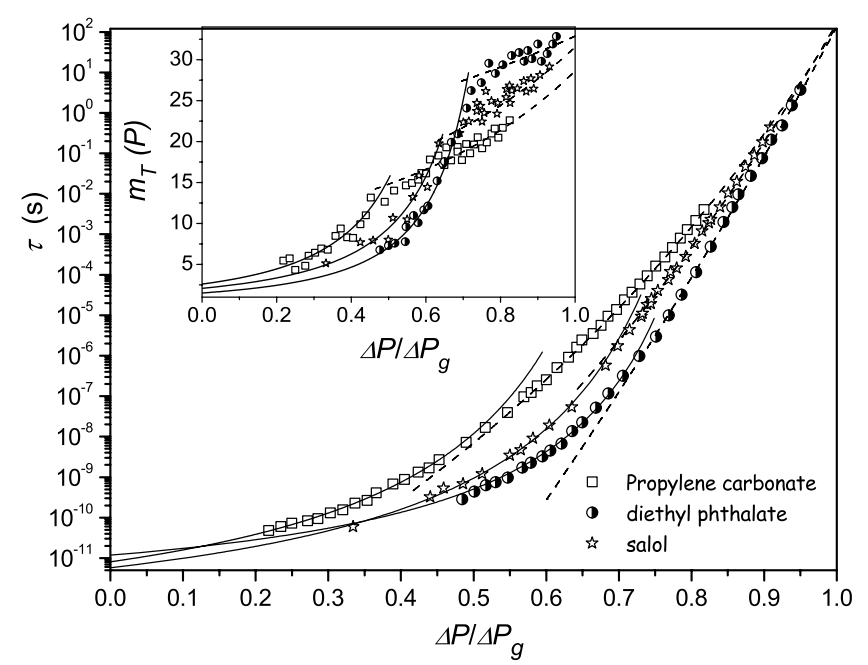

Figure 9. A possible pressure counterpart of the Angell plot, based on experimental data for the isothermic, pressure evolution of dielectric relaxation time for diethyl phthalate $(T \approx 293 \mathrm{~K}[23,41])$, propylene carbonate $(T \approx 273 \mathrm{~K}[29])$ and salol $(T \approx 343 \mathrm{~K}[19])$; $\Delta P=P-P_{S}, \Delta P_{\mathrm{g}}=P_{\mathrm{g}}-P_{S}$ and $P_{S}<0$ are estimates for the stability limit for the given liquid. Solid and dashed curves are for PVFT (equation (11)), portraying experimental data in subsequent dynamical domains. The experimental data presented are for $P>0.1 \mathrm{MPa}$ but the novel PVFT-based parameterizations (equation (11), the solid curve) make an extension into the negative pressures domain possible. The inset shows the pressure evolution of the modified steepness index $m_{T}(P)=\mathrm{d} \log _{10} \tau / \mathrm{d}\left(\Delta P / \Delta P_{\mathrm{g}}\right)$, for experimental data and curves given from the main part of the plot.

steepness index introduced by equation (22): $m_{T}(P)=$ $\log _{10} \Delta P / \Delta P_{\mathrm{g}}=\log _{10}\left[\left(P-P_{\mathrm{SL}}\right) /\left(P_{\mathrm{g}}-\pi\right)\right] . \quad$ It collects $\tau(P)$ data for DEP, PC, and salol. For the latter, data were obtained via the scaling of $\tau(P)$ and $\eta(P)$ dependences reported in [19]. For salol the analysis via the PVFT equation (11) yielded: $D_{P}=4.5, P_{0}=0.84 \mathrm{GPa}, \tau_{0}^{P}=5 \mathrm{ps}$ for $P<P_{B}$ and $D_{P}=90, P_{0}=2.9 \mathrm{GPa}, \tau_{0}^{P}=0.5 \mu \mathrm{s}$ for $P>P_{B}$.

The inset in figure 9 shows the derivative of scaled data from the main part of the plot, i.e. for the pressure evolution of the steepness index $m_{T}(P)$ linked to equation (22): the solid and the dashed curves are for subsequent dynamical domains, in agreement with the main part of figure 9. When discussing results presented in figure 9 we would like to indicate the following issues.

(i) The derivative-based plot of scaled experimental data, $m_{T}=\operatorname{dlog}_{10} \tau / \mathrm{d}\left(\Delta P / \Delta P_{\mathrm{g}}\right)$ versus $\Delta P / \Delta P_{\mathrm{g}}$, shows clear hallmarks of a change in dynamics at $\tau\left(P_{B}\right) \sim$ $10^{-7} \mathrm{~s}$. This may suggest that the dynamic crossover is not an artifact associated with using VFT or PVFT parameterization of data but a general feature of vitrifying systems.

(ii) Although all compounds in figure 9 exhibit a nonlinear (non-Arrhenius) evolution of relaxation times, for selected ranges of pressures the almost Arrhenius description seems to offer a reasonable description. This may be the case for the high pressure dynamical domain $\left(P_{B}<\right.$ $P<P_{\mathrm{g}}$ ), where large values of the steepness index $m_{T}$ are associated with relatively large values of the fragility strength coefficient $D_{P}$. Such a situation may occur also far away from the vitrification point, for $\Delta P / \Delta P_{\mathrm{g}} \rightarrow 0$, where very small values of $m_{T}$ are linked to small or relatively small values of $D_{P}$. The latter may be expected in the negative pressures domain.

(iii) A marked non-Arrhenius behavior occurs for $P \rightarrow P_{B}$.

(iv) One may expect all issues indicated above to be valid also for the basic (temperature-related) Angell plot, when recalling the equivalence of $m_{T}$ and $m_{P}$ steepness indexes obtained above.

Both $D_{T}$ and $D_{P}$ values are constant in the given dynamical domain and seem to increase when shifting into the dynamical domain in the immediate vicinity of the glass transition point $\left(T_{\mathrm{g}}, P_{\mathrm{g}}\right)$ [5, 9-14, 17-26, 41-45]. Large values of $D_{T}$ and $D_{P}$ reflect relatively small increases of $m_{P}$ and $m_{T}$ steepness indexes on cooling or pressurizing and, vice versa, small values $D_{T}$ and $D_{P}$ reflect significant increases of $m_{P}$ and $m_{T}$ on cooling or pressurizing.

\section{Conclusions}

Herein we have analyzed the pressure evolution of dynamic properties of supercooled liquids, using VFT-type and Avramov-model-type expressions. A modified pressure counterpart of the VFT expression (equation (8)) yields more reliable determinations of the fragility strength coefficient $D_{P}$, and also makes it possible to estimate the loci of the stability limit at negative pressures. Estimations of this coefficient, so far based on direct measurements, are extremely difficult experimental tasks [75-81]. The novel PVFT equation enabled a qualitatively revised answer to the question 'Does fragility depend on pressure?' [13] to be obtained; namely it leads to the equivalence of $m_{P}$ and $m_{T}$ steepness indexes and related fragilities. All of this led to a proposal for a pressure counterpart of the Angell plot, in the opinion of the authors totally isomorphic to the basic, temperature-related Angell plot.

The comparison of the Avramov-type (AvM) and VFT/PVFT descriptions led to the conclusion that the latter seems to offer a better parameterization of experimental data. However, only for the AvM-type equation does a single set of parameters seem to be sufficient for portraying the extensive set of $\tau(T)$ or $\tau(P)$ data. This can be associated with the lack of hallmarks of dynamical crossover for such parameterization. Nevertheless, small but permanent distortions between the AvM parameterization and experimental data seem to occur. They are particularly visible for the derivative-based, distortion-sensitive analysis shown in the insets in figures $1,3,4$ and 6 . Notwithstanding this, the analysis of a single set of $(\tau(T), \tau(P))$ via the AvM-type description (equation (16)), supported by derivative analysis (equation (17)), can yield reliable estimates of $\pi$ and $b$ coefficients, enabling the reproducing of the $T_{\mathrm{g}}(P)$ evolution. All of this can indicate the AvM-based description to be interesting tool for practical and technological applications.

In conclusion, this paper has presented the discussion of a judicious selection of formulations used to describe the 
evolution of dynamic properties as a function of temperature and pressure, supported by derivative-based and distortionsensitive analysis. Particularly worth stressing is the inclusion of the negative pressure domain, hardly discussed in such contexts so far.

\section{Acknowledgments}

We thank the US Office of Naval Research for support. We would also like to acknowledge the support of the CLG NATO (ref. CBP. NUKR.CLG 982312). Finally, some parts of these studies were possible due to the help of the Ministry of Science and Higher Education (Poland, grant ref. N202 147 32/4240)

\section{References}

[1] Donth E 1998 The Glass Transition. Relaxation Dynamics in Liquids and Disordered Material (Springer Series in Material Sci. II vol 48) (Berlin: Springer)

[2] Dyre J C 2006 Rev. Mod. Phys. 78953

[3] Angell C A 1995 Proc. Natl Acad. Sci. 926675

[4] Floudas G 2004 Prog. Polym. Sci. 291143

[5] Roland C M, Hensel-Bielowka S, Paluch M and Casalini R 2005 Rep. Prog. Phys. 681405

[6] Greaves G N and Sen S 2007 Adv. Phys. 561

[7] Paluch M, Rzoska S J, Habdas P and Zioło J 1998 J. Phys.: Condens. Matter 104131

[8] Andersson S P and Andersson O 1998 Macromolecules 312999

[9] Paluch M, Hensel-Bielowka S and Zioło J 1999 J. Chem. Phys. 11010978

[10] Paluch M, Hensel-Bielowka S and Psurek T 2000 J. Chem. Phys. 1134374

[11] Blumer Ch and Mader K 2005 Pharm. Res. 221708

[12] Paluch M, Ngai K L and Hensel-Bielowka S 2001 J. Chem. Phys. 11410872

[13] Paluch M, Gapiński J, Patkowski A and Fischer E W 2001 J. Chem. Phys. 1148048

[14] Pawlus S, Paluch M, Sekula M, Ngai K L, Rzoska S J and Zioło J 2003 Phys. Rev. E 68021503

[15] Rao K J, Bhat M H and Kumar S 2001 J. Indian Inst. Sci. 813

[16] Suzuki A, Masuko M and Wakisaka K 2002 Tribol. Int. 3555

[17] Casalini R, Paluch M and Roland C M 2003 J. Chem. Phys. 1185701

[18] Casalini R and Roland C M 2003 J. Chem. Phys. 11911951

[19] Casalini R, Paluch M and Roland C M 2003 J. Phys. Chem. 1072369

[20] Patkowski A, Paluch M and Gapiński J 2003 J. Non-Cryst. Solids 330259

[21] Paluch M, Roland C M, Gapinski J and Patkowski A 2003 J. Chem. Phys. 1183177

[22] Paluch M and Roland C M 2003 J. Non-Cryst. Solids 316413

[23] Pawlus S, Paluch M, Sekuła M, Ngai K L and Zioło J 2003 Phys. Rev. E 68021503

[24] Behrens H and Schulze F 2003 Am. Mineral. 881351

[25] Mpoukouvalas K and Floudas G 2003 Phys. Rev. E 68031801

[26] Patkowski A, Gapiński J and Meier G 2004 Colloid Polym. Sci. 282874

[27] Casalini R, Paluch M, Psurek T and Roland C M 2004 J. Mol. Liq. 11153

[28] Casalini R and Roland C M 2004 Phys. Rev. Lett. 92245702

[29] Pawlus S, Casalini R, Roland C M, Paluch M, Rzoska S J and Zioło J 2004 Phys. Rev. E 70061501

[30] Reiser A, Kasper G and Hunklinger S 2004 Phys. Rev. Lett. 92125701
[31] Papadopopulos P, Floudas G, Schnell I, Klok H A, Aliferis T, Iatrou H and Hadjichristidis N $2005 \mathrm{~J}$. Chem. Phys. 122224906

[32] Casalini R and Roland C M 2005 Phys. Rev. B 71014210

[33] Prevosto D, Capaccioli S, Lucchesi M, Rolla P A, Paluch M, Pawlus S and Zioło J 2005 J. Chem. Phys. 122061102

[34] Casalini R and Roland C M 2004 Phys. Rev. E 69062501

[35] Casalini R and Roland C M 2005 Phys. Rev. B 71014210

[36] Reiser A, Kasper G and Hunklinger S 2005 Phys. Rev. B 72094204

[37] Drozd-Rzoska A 2005 Phys. Rev. E 72041505

[38] Kriegs H, Gapiński J, Meier G, Paluch M, Pawlus S and Patkowski A 2006 J. Chem. Phys. 124104901

[39] Reiser A and Kasper G 2006 Europhys. Lett. 761137

[40] Prevosto D, Capaccioli S, Lucchesi M, Rolla P A, Paluch M and Pawlus S 2006 Phys. Rev. E 73104205

[41] Drozd-Rzoska A and Rzoska S J 2006 Phys. Rev. E 73041502

[42] Drozd-Rzoska A, Rzoska S J, Pawlus S and Tamarit J Ll 2006 Phys. Rev. B 73224205

[43] Drozd-Rzoska A, Rzoska S J, Pawlus S and Tamarit J L1 2006 Phys. Rev. B 74064201

[44] Grzybowska K, Pawlus S, Mierzwa M and Paluch M 2006 J. Chem. Phys. 125144507

[45] Mpoukouvalas K, Gomopoulos N, Floudas G, Herrmann C, Hanewald A and Best A 2006 Polymer 477170

[46] Win K Z and Menon N 2006 Phys. Rev. E 73040501

[47] Avramov I 2007 J. Volcanol. Geotherm. Res. 160579

[48] Kalakkunnath S, Kalika D S, Lin H Q, Raharjo R D and Freeman B D 2007 Polymer 48579

[49] Drozd-Rzoska A, Rzoska S J, Paluch M, Imre A R and Roland C M 2007 J. Chem. Phys. 126164504

[50] Ahmed A J, Prabhu S T, Raghavan G S V and Ngadi M 2007 J. Food Eng. 791207

[51] Vogel H 1921 Phys. Z. 22645

Fulcher G S 1925 J. Am. Chem. Soc. 8339

Tammann G and Hesse W 1926 Z. Anorg. Allg. Chem. 156245

[52] Johari G P 2006 Phil. Mag. 861567

[53] Cook R L, King H R, Herbst C A and Herschabach D R 1994 J. Chem. Phys. 1005178

[54] Bendler J T, Fontanella J J, Shlesinger M F and Wintesgill M C 2001 Electrochim. Acta $\mathbf{4 6} 1615$

[55] Faupel F and Franck W 2003 Rev. Mod. Phys. 75237

[56] Leyser H, Schulte A, Doster W and Petry W 1995 Phys. Rev. E 515899

[57] Dreyfus A, Aouadi A, Gapiński J, Matos-Lopes M, Steffen W, Patkowski A and Pick R M 2003 Phys. Rev. E 68011204

[58] Greet R J and Turnbull D 1967 J. Chem. Phys. 461243

[59] Turnbull D and Cohen M H 1961 J. Chem. Phys. 311164

[60] Doolittle A K and Doolittle D B 1957 J. Appl. Phys. 28901

[61] Johari G P and Whalley E 1972 Faraday Symp. Chem. Soc. 623

[62] Paluch M, Zioło J, Rzoska S J and Habdas P 1996 Phys. Rev. E 544008

[63] Walther C 1931 Erdöl Teer 7382

[64] Harrison G 1976 The Dynamic Properties of Supercooled Liquids (New York: Academic)

[65] Bässler H 1987 Phys. Rev. Lett. 58767

[66] Avramov I 1998 Glastech. Ber. C 71198

[67] Zhang Y, Xu Z and Liu Y 2003 Am. Mineral. 881741

[68] Avramov I 2000 J. Non-Cryst. Solids 262258

[69] Avramov I 2005 J. Non-Cryst. Solids 3513163

[70] Puzenko A, Ishai P B and Paluch M 2007 J. Chem. Phys. 127094503

[71] Drozd-Rzoska A, Rzoska S J and Imre A R 2007 J. Non-Cryst. Solids 3533915

[72] Casalini R, Mohanty U and Roland C M 2006 J. Chem. Phys. 125014505

[73] Ojovan M I and Lee W E 2005 Phys. Chem. Glasses 467

[74] Casalini R and Roland C M 2007 J. Non-Cryst. Solids 3533936 
[75] Angell C A and Quing Z 1989 Phys. Rev. B 398784

[76] Imre A R, Maris H J and Williams P R (ed) 2001 Liquids under Negative Pressures (NATO Sci. Series II vol 84) (Dordrecht: Kluwer)

[77] Speedy R J 2004 J. Chem. Phys. 12010182

[78] Sastry S 2000 Phys. Rev. Lett. 85590

[79] Landa L and Landa K 2004 J. Non-Cryst. Solids 34859

[80] Debenedetti P G and Stanley H E 2003 Phys. Today 5640

[81] Utz M, Debenedetti P G and Stillinger F H 2001 J. Chem. Phys. 11410049

[82] Angell C A 1985 Relaxations in Complex Systems ed K L Ngai (Washington DC: NRL) p 3

[83] Böhmer R, Ngai K L, Angell C A and Plazek D J 1993 J. Chem. Phys. 994201

[84] Dreyfus C, Le Grand A, Gapiński J, Steffen W and Patkowski A 2004 Eur. Phys. J. B 42309

[85] Alba-Simionesco Ch and Tarjus G 2006 J. Non-Cryst. Solids 3524888
[86] Casalini R, Mohanty U and Roland C M 2006 J. Chem. Phys. 125014505

[87] Stickel F, Fischer E W and Richert R 1996 J. Chem. Phys. 1042043

[88] Hansen C, Stickel F, Berger P, Richert R and Fischer E W 1997 J. Chem. Phys. 1071086

[89] Novikov V N and Sokolov A P 2003 Phys. Rev. E. 67031507

[90] Rebelo L P N 1999 Phys. Chem. Chem. Phys. 14277

[91] Imre A R, Melnichenko G and van Hook W A 1999 Phys. Chem. Chem. Phys. 14287

[92] Deb S K, Wilding M, Somayazulu M and McMillan P F 2001 Nature 414528

[93] Yu L 2001 Adv. Drug. Deliv. Rev. 4827

[94] Craig D Q M, Royall P G, Kett V L and Hopton M L 1999 Int. J. Pharmaceut. 179179

[95] Paluch M, Grzybowska K and Grzybowski A 2007 J. Phys.: Condens. Matter 19205117

[96] Brenskelle L A and McCoy B 2006 J. Chem. Phys. 124084502 